\title{
COMMUTING FULLY INVARIANT CONGRUENCES ON FREE COMPLETELY REGULAR SEMIGROUPS
}

\author{
F. PASTIJN
}

\begin{abstract}
We show that "almost all" fully invariant congruences on every free completely regular semigroup commute. From this it is shown that the lattice of completely regular semigroup varieties is arguesian.
\end{abstract}

\section{INTRODUCTION AND SUMMARY}

A completely regular semigroup is a semigroup which is a union of groups. If $S$ is a completely regular semigroup and $a \in S$, then $a$ belongs to a unique maximal subgroup $H_{a}$ of $S$; we denote by $a^{0}$ the identity element of $H_{a}$ and by $a^{-1}$ the inverse of $a$ in $H_{a}$. Obviously a completely regular semigroup can be viewed as an algebra of type $\langle 2,1\rangle$, where ${ }^{-1}$ is the unary operation. Completely regular semigroups satisfy, apart from the associative law, the identities

$$
\left(x^{-1}\right)^{-1}=x, \quad x x^{-1}=x^{-1} x, \quad x x^{-1} x=x .
$$

Conversely, if $S$ belongs to the variety $\mathbf{C R}$ consisting of the algebras of type $\langle 2,1\rangle$ satisfying the associative law and the identities (1), then $S$ is a completely regular semigroup and for $a \in S, a^{-1}$ is the inverse of $a$ within the maximal subgroup of $S$ to which $a$ belongs. We shall therefore consider the class of completely regular semigroups to be a variety of algebras of type $\langle 2,1\rangle$ satisfying the associative law and the identities (1). In the presence of the identities (1) we shall use the notation $x^{0}=x x^{-1}=x^{-1} x$. We denote by $\mathbf{U}$ the variety of algebras of type $\langle 2,1\rangle$ where the associative law holds. The algebras of $\mathbf{U}$ will be called unary semigroups. Thus, $\mathbf{C R}$ is a subvariety of $\mathbf{U}$.

A band is a semigroup in which every element is an idempotent. Thus a band is a union of trivial groups and the class of bands is a subvariety $\mathbf{B}$ of CR consisting of the unary semigroups satisfying, apart from the associative law, the identities

$$
x^{2}=x, \quad x^{-1}=x .
$$

The class SL of commutative bands forms a subvariety of $\mathbf{B}$ and a member of SL will be called a semilattice. The variety CS of completely simple semigroups

Received by the editors December 1, 1988 and, in revised form, October 5, 1989.

1980 Mathematics Subject Classification (1985 Revision). Primary 20M05, 20M07; Secondary 06C05, 08B15, 08B10. 
is the subvariety of $\mathbf{C R}$ consisting of the completely regular semigroups which satisfy the additional identity

$$
(x y x)^{0}=x^{0}
$$

The variety $\mathbf{G}$ of groups is the subvariety of $\mathbf{C S}$ which satisfies the additional identity

$$
x^{0}=y^{0}
$$

An identity $p=q$ of type $\langle 2,1\rangle$ will be called regular if the set of variables which occur in $p$ coincides with the set of variables which occur in $q$. One can easily show that a subvariety $\mathbf{V}$ of $\mathbf{C R}$ contains $\mathbf{S L}$ if and only if every identity satisfied by all the members of $\mathbf{V}$ is regular. If all the members of $\mathbf{V}$ satisfy some nonregular identity, then SL $\nsubseteq \mathbf{V}$ and it now easily follows from [14, IV.2] that $\mathbf{V} \subseteq \mathbf{C S}$. In other words, the lattice $\mathscr{L}(\mathbf{C R})$ of subvarieties of $\mathbf{C R}$ is the disjoint union of the two intervals [T, CS] and [SL, CR], T being the least element of $\mathscr{L}(\mathbf{C R})$.

Let $X$ be a countable set and $F_{X}(\mathbf{U})$ the free unary semigroup on $X$. If $\mathbf{V}$ is a subvariety of $\mathbf{U}$ then the least congruence $\rho$ on $F_{X}(\mathbf{C R})$, such that $F_{X}(\mathbf{U}) / \rho \in \mathbf{V}$ is a fully invariant congruence of $F_{X}(\mathbf{U})$, which will be denoted by $\rho_{\mathbf{V}}$. If $\rho$ is a fully invariant congruence on $F_{X}(\mathbf{U})$, then the variety generated by $F_{X}(\mathbf{U}) / \rho$ will be denoted by $\mathbf{V}_{\rho}$. For any completely regular semigroup $S$, let $C(S)$ be the lattice of fully invariant congruences on $S$. Let us denote by $\left[\rho_{\mathbf{C R}}\right)$ the dual ideal of $C\left(F_{X}(\mathbf{U})\right)$ generated by $\rho_{\mathbf{C R}}$. It is well known that

$$
\mathscr{L}(\mathbf{C R}) \rightarrow\left[\rho_{\mathbf{C R}}\right), \quad \mathbf{V} \rightarrow \rho_{\mathbf{V}}
$$

and

$$
\left[\rho_{\mathbf{C R}}\right) \rightarrow \mathscr{L}(\mathbf{C R}), \quad \rho \rightarrow \mathbf{V}_{\rho},
$$

are mutually inverse dual isomorphisms. From the above it now follows that $\left[\rho_{\mathbf{C R}}\right)$ is the disjoint union of the two intervals $\left[\rho_{\mathbf{C S}}, \rho_{\mathbf{T}}\right]$ and $\left[\rho_{\mathbf{C R}}, \rho_{\mathbf{S L}}\right]$.

In this paper we shall show that for every completely simple semigroup $S$, $C(S)$ is a lattice of commuting congruences. As a consequence the interval $\left[\rho_{\mathrm{CS}}, \rho_{\mathrm{T}}\right]$ is a lattice of commuting congruences. We shall also show that the interval $\left[\rho_{\mathbf{C R}}, \rho_{\mathbf{S L}}\right]$ is a lattice of commuting congruences. Thus $\left[\rho_{\mathbf{C R}}, \rho_{\mathbf{S L}}\right]$ and $\left[\rho_{\mathbf{C S}}, \rho_{\mathrm{T}}\right]$ are linear in the sense of [6]. However, as we shall show, it is not true that every element of $\left[\rho_{\mathbf{C S}}, \rho_{\mathrm{T}}\right]$ commutes with every element of $\left[\rho_{\mathbf{C R}}, \rho_{\mathbf{S L}}\right]$. Nevertheless the above results together with the fact that $\mathbf{S L}$ is a neutral element in $\mathscr{L}(\mathbf{C R})$ [7] entail that $\mathscr{L}(\mathbf{C R})$ is arguesian by [9]. In particular, $\mathscr{L}(\mathbf{C R})$ is modular. The same result is obtained in [12], the proof of which is based on the more involved main theorem of [17].

In the following we shall use without further notice the terminology, notation and basic results of $[3,14,15]$. 
2. The FUlly INVARIANT CONGRUENCES ON $F_{X}(\mathbf{C R})$ DO NOT COMMUTE

If $\mathbf{V}$ is any subvariety of $\mathbf{C R}$, then we can identify $F_{X}(\mathbf{U}) / \rho_{\mathbf{V}}$ with the free object $F_{X}(\mathbf{V})$ in $\mathbf{V}$ on the set $X$. Thus the elements of $F_{X}(\mathbf{V})$ are of the form $u \rho_{\mathbf{V}}, u \in F_{X}(\mathbf{U})$. In order to avoid this cumbersome notation we shall write $u$ instead of $u \rho_{\mathbf{V}}$. We recall that for any $u \in F_{X}(\mathbf{V})$ the subgroup $\langle u\rangle$ generated by $u$ consists of the elements $u^{n}, n \in \mathbf{Z}$; the notation $u^{n}, n \in \mathbf{Z}$, is now perfectly unambiguous.

We start with the negative result announced above. The following was suggested to us by M. V. Volkov.

Proposition 1. Let $\tau_{\mathbf{G}}$ and $\tau_{\mathbf{S L}}$ be the least group and the least semilattice congruence on $F_{X}(\mathbf{C R})$. Then $\tau_{\mathbf{G}} \circ \tau_{\mathbf{S L}} \neq \tau_{\mathbf{S L}} \circ \tau_{\mathbf{G}}$.

Proof. Let $x$ and $y$ be different elements of $X$. Then

$$
x^{0} \tau_{\mathbf{G}} y^{0} \tau_{\mathbf{S L}} y^{n}
$$

for every $n \in \mathbf{Z}$ gives $\langle y\rangle \subseteq x^{0}\left(\tau_{\mathrm{G}} \circ \tau_{\mathrm{SL}}\right)$. On the other hand $x^{0} \tau_{\mathrm{SL}}=\langle x\rangle$ and $x^{m} \tau_{\mathbf{G}} y^{n} \Leftrightarrow m=n=0$ imply that

$$
\langle y\rangle \cap x^{0}\left(\tau_{\mathbf{S L}} \circ \tau_{\mathbf{G}}\right)=\left\{y^{0}\right\} .
$$

Hence

$$
x^{0}\left(\tau_{\mathbf{G}} \circ \tau_{\mathbf{S L}}\right) \neq x^{0}\left(\tau_{\mathbf{S L}} \circ \tau_{\mathbf{G}}\right)
$$

and $\tau_{\mathbf{G}}$ and $\tau_{\mathbf{S L}}$ do not commute.

Since $\rho_{\mathbf{G}} \in\left[\rho_{\mathbf{C S}}, \rho_{\mathbf{T}}\right]$ and $\rho_{\mathbf{S L}} \in\left[\rho_{\mathbf{C R}}, \rho_{\mathbf{S L}}\right]$ the foregoing implies that the fully invariant congruences in $\left[\rho_{\mathrm{CS}}, \rho_{\mathrm{T}}\right]$ do not necessarily commute with the fully invariant congruences of $\left[\rho_{\mathbf{C S}}, \rho_{\mathbf{S L}}\right]$.

\section{FULLY INVARIANT CONGRUENCES ON COMPLETELY SIMPLE SEMIGROUPS COMMUTE}

Let $S$ be a completely regular semigroup and $\rho$ a congruence relation on $S$. The set of idempotents of $S$ will be denoted by $E(S)$. Then

$$
\operatorname{tr} \rho=\rho \cap(E(S) \times E(S))
$$

and

$$
\operatorname{ker} \rho=\left\{a \in S \mid a \rho a^{0}\right\} \text {. }
$$

For congruences $\rho$ and $\theta$ on $S$ we put

$$
\rho K \theta \Leftrightarrow \operatorname{ker} \rho=\operatorname{ker} \theta .
$$

Proposition 2. For congruences $\rho$ and $\theta$ on a completely regular semigroup $S$ we have

(i) $\rho K \theta \Leftrightarrow \rho \cap \mathscr{H}=\theta \cap \mathscr{H}$.

(ii) $((\rho \cap \mathscr{D}) \vee(\theta \cap \mathscr{D})) \cap \mathscr{H}=(\rho \cap \mathscr{H}) \circ(\theta \cap \mathscr{H})=(\theta \cap \mathscr{H}) \circ(\rho \cap \mathscr{H})$. 
Proof. (i) Since $a \mathscr{H} a^{0}$ for every $a \in S$ we have that

$$
\operatorname{ker} \rho=\left\{a \in S \mid a(\rho \cap \mathscr{H}) a^{0}\right\},
$$

hence

$$
\rho \cap \mathscr{H}=\theta \cap \mathscr{H} \Rightarrow \operatorname{ker} \rho=\operatorname{ker} \theta .
$$

Let $a, b \in S$ such that $a \mathscr{H} b$ and $a b^{-1} \in \operatorname{ker} \rho$. Then $a b^{-1} \rho\left(a b^{-1}\right)^{0}=$ $a^{0}=b^{0}$ so that

$$
a=a b^{0}=a b^{-1} b \rho b^{0} b=b
$$

and $a(\rho \cap \mathscr{H}) b$. Therefore

$$
\operatorname{ker} \rho=\operatorname{ker} \theta \Rightarrow \rho \cap \mathscr{H}=\theta \cap \mathscr{H}
$$

and this in combination with (7) yields (i).

(ii) If $c \in S$ such that $a(\rho \cap \mathscr{H}) c(\theta \cap \mathscr{H}) b$ then

$$
c^{-1} b(\theta \cap \mathscr{H}) b^{0}=a^{0}, \quad a c^{-1}(\rho \cap \mathscr{H}) a^{0}=b^{0} ;
$$

thus $a(\theta \cap \mathscr{H}) a c^{-1} b(\rho \cap \mathscr{H}) b$ and so $(\rho \cap \mathscr{H}) \circ(\theta \cap \mathscr{H}) \subseteq(\theta \cap \mathscr{H}) \circ(\rho \cap \mathscr{H})$. By symmetry the equality

$$
(\rho \cap \mathscr{H}) \circ(\theta \cap \mathscr{H})=(\theta \cap \mathscr{H}) \circ(\rho \cap \mathscr{H})
$$

prevails. It goes without saying that $(8)$ is the join $(\rho \cap \mathscr{H}) \vee(\theta \cap \mathscr{H})$ of $\rho \cap \mathscr{H}$ and $\theta \cap \mathscr{H}$ in the lattice $\mathrm{Eq}(S)$ of equivalences on $S$. Hence

$$
(\rho \cap \mathscr{H}) \circ(\theta \cap \mathscr{H}) \subseteq((\rho \cap \mathscr{D}) \vee(\theta \cap \mathscr{D})) \cap \mathscr{H}
$$

is obviously satisfied.

For every $a \in S, D_{a}$ is a completely simple semigroup and so $\rho \cap\left(D_{a} \times D_{a}\right)$ and $\theta \cap\left(D_{a} \times D_{a}\right)$ are congruences on $D_{a}$. From the description of the lattice of congruences on a completely simple semigroup [3,10.7] it follows that for any $\mathscr{H}$-class $H$ contained in $D_{a}$,

$$
\begin{aligned}
& (\rho \cap(H \times H)) \vee(\theta \cap(H \times H)) \\
& \quad=\left(\left(\rho \cap\left(D_{a} \times D_{a}\right)\right) \vee\left(\theta \cap\left(D_{a} \times D_{a}\right)\right)\right) \cap(H \times H) .
\end{aligned}
$$

Hence, putting

$$
\gamma=\bigcup_{a \in S}\left(\left(\rho \cap\left(D_{a} \times D_{a}\right)\right) \vee\left(\theta \cap\left(D_{a} \times D_{a}\right)\right)\right)
$$

we have that

$$
\begin{aligned}
\gamma \cap \mathscr{H} & =(\rho \cap \mathscr{H}) \vee(\theta \cap \mathscr{H})=(\rho \cap \mathscr{H}) \circ(\theta \cap \mathscr{H}) \\
& =(\theta \cap \mathscr{H}) \circ(\rho \cap \mathscr{H})
\end{aligned}
$$

in the lattice $\operatorname{Eq}(S)$. It now suffices to show that $\gamma=(\rho \cap \mathscr{D}) \vee(\theta \cap \mathscr{D})$ in the lattice of congruences on $S$. For this it suffices to show that the equivalence $\gamma$ of (10) is actually a congruence on $S$. 
Let $a \gamma b$ for some $a, b \in S$. Then there exists a positive integer $n$ and $d_{0}, \ldots, d_{n} \in D=D_{a}=D_{b}$ such that

$$
a=d_{0}(\rho \cap(D \times D)) d_{1}(\theta \cap(D \times D)) \cdots d_{n-1}(\theta \cap(D \times D)) d_{n}=b .
$$

For $c \in S$ and $D^{\prime}=D_{a c}=D_{b c}$ we then have

$$
a c=d_{0} c\left(\rho \cap\left(D^{\prime} \times D^{\prime}\right)\right) d_{1} c \cdots d_{n-1} c\left(\theta \cap\left(D^{\prime} \times D^{\prime}\right)\right) d_{n} c=b c,
$$

whence $a c \gamma b c$. A dual argument shows that $c a \gamma c b$. Thus, $\gamma$ is a congruence and (ii) holds.

Proposition 3. Let $\rho$ and $\theta$ be congruences on the completely regular semigroup $S$ such that $\rho, \theta \subseteq \mathscr{D}$. If $\operatorname{tr} \rho$ and $\operatorname{tr} \theta$ commute, then $\rho \circ \theta=\theta \circ \rho$. In particular, if $\operatorname{tr} \rho \subseteq \operatorname{tr} \theta$, then $\rho \circ \theta=\theta \circ \rho$.

Proof. Let $a, b, c \in S$ such that $a \theta c \rho b$. Then $a^{0} \theta c^{0} \rho b^{0}$ and since $\operatorname{tr} \rho$ commutes with $\operatorname{tr} \theta$, we find that $a^{0} \rho d^{0} \theta b^{0}$ for some $d \in S$. Since $\rho, \theta \subseteq \mathscr{D}$ we have that the elements $a, b, c, d$ are $\mathscr{D}$-related in $S$, and consequently also that

$$
d^{0} a d^{0}((\rho \vee \theta) \cap \mathscr{H}) d^{0} b d^{0} .
$$

Using Proposition 2, we know that there exists an element $e \in S$ such that

$$
d^{0} a d^{0}(\rho \cap \mathscr{H}) e(\theta \cap \mathscr{H}) d^{0} b d^{0} .
$$

Therefore

$$
a=a^{0} a a^{0} \rho d^{0} a d^{0} \rho e \theta d^{0} b d^{0} \theta b^{0} b b^{0}=b .
$$

We proved that $\theta \circ \rho \subseteq \rho \circ \theta$. By symmetry the equality prevails.

Theorem 4. The fully invariant congruences on a completely simple semigroup commute.

Proof. Let $S$ be a completely simple semigroup and $\rho$ a fully invariant congruence on $S$. For $e, f \in S$, the mapping

$$
\begin{aligned}
\phi: S \rightarrow S, & a \rightarrow a^{0} \quad \text { if } a \mathscr{L} e, \\
a & \rightarrow(a f)^{0} \quad \text { otherwise }
\end{aligned}
$$

is an endomorphism of $S$. From this it follows that either

$$
(\rho \cap \mathscr{R}) \cap(E(S) \times E(S)) \text { is the equality on } E(S)
$$

or

$$
(\rho \cap \mathscr{R}) \cap(E(S) \times E(S))=\mathscr{R} \cap(E(S) \times E(S)) .
$$

From this and its dual we have that the following four possibilities may occur: either

(i) $\operatorname{tr} \rho$ is the equality on $E(S)$,

(ii) $\operatorname{tr} \rho=\mathscr{L} \cap(E(S) \times E(S))$,

(iii) $\operatorname{tr} \rho=\mathscr{R} \cap(E(S) \times E(S))$, or

(iv) $\operatorname{tr} \rho=E(S) \times E(S)$. 
Now let $\rho$ and $\theta$ be fully invariant congruences on $S$. If $\operatorname{tr} \rho \subseteq \operatorname{tr} \theta$ or $\operatorname{tr} \theta \subseteq \operatorname{tr} \rho$ then $\rho \circ \theta=\theta \circ \rho$ by Proposition 3. If this is not the case, then we can assume without loss of generality that $\operatorname{tr} \rho=\mathscr{L} \cap(E(S) \times E(S))$ and $\operatorname{tr} \theta=$ $\mathscr{R} \cap(E(S) \times E(S))$. Then $\rho \subseteq \mathscr{L}$ and $\theta \subseteq \mathscr{R}$ so that $\rho \circ \theta=\theta \circ \rho$ by [10].

Corollary 5. The sublattice $\left[\rho_{\mathrm{CS}}, \rho_{\mathrm{T}}\right]$ of the lattice $C\left(F_{X}(\mathbf{U})\right)$ of fully invariant congruences on $F_{X}(\mathbf{U})$ consists of commuting congruences. The lattice $\mathscr{L}(\mathbf{C S})$ of subvarieties of $\mathbf{C S}$ is arguesian.

Proof. Let $F_{X}(\mathbf{C S})$ be the free completely simple semigroup on the set $X$. We may identify $F_{X}(\mathbf{C S})$ with $F_{X}(\mathbf{U}) / \rho_{\mathbf{C S}}$. By Theorem 4 the fully invariant congruences on $F_{X}(\mathbf{C S})$ commute. The mapping

$$
\left[\rho_{\mathbf{C S}}, \rho_{\mathbf{T}}\right] \rightarrow C\left(F_{X}(\mathbf{C S})\right), \quad \rho \rightarrow \rho / \rho_{\mathbf{C S}},
$$

is an isomorphism of lattices and we infer that the congruences in $\left[\rho_{\mathbf{C S}}, \rho_{\mathbf{T}}\right]$ commute. Since $\mathscr{L}(\mathbf{C S})$ is dually isomorphic with $\left[\rho_{\mathbf{C S}}, \rho_{\mathbf{T}}\right]$ we have that $\mathscr{L}(\mathbf{C S})$ is arguesian.

From the above it follows that $\mathscr{L}(\mathbf{C S})$ is modular. This result was also proved in [7].

\section{4. $\mathscr{L}(\mathbf{C R})$ IS ARGUESIAN}

The free unary semigroup $F_{X}(\mathbf{U})$ can be considered to be a subset of the free monoid $M$ on the set $X \cup\left\{(,)^{-1}\right\}$. For $u \in F_{X}(\mathbf{U})$ with $u=v w$ in $M$ we say that $v$ is an initial segment of $u$ and $w$ is a final segment of $u$. We then denote by $\hat{v}$ and $\hat{w}$ the elements of $M$ which are obtained from $v$ and $w$ by deleting all unmatched parentheses. Further $c(u)$, called the content of $u$, is the set of all variables from $X$ which occur in $u$. For $u \in M$ and $|c(u)|>1$ we put $s(u)=\hat{v}$, where $v$ is the largest initial segment of $u$ such that $|c(v)|=|c(u)|-1$, and we define $\bar{s}(u)$ by

$$
c(u) \backslash c(s(u))=\{\bar{s}(u)\} .
$$

Dually $d(u)=\hat{w}$, where $w$ is the largest final segment of $u$ such that $|c(w)|=$ $|c(u)|-1$, and we define $\bar{d}(u)$ by

$$
c(u) \backslash c(d(u))=\{\bar{d}(u)\} .
$$

If $u \in M$ with $c(u) \leq 1$, then we assume $s(u)=d(u)$ to be the identity $\lambda$ of $M$. The transformations $s$ and $d$ generate a subsemigroup of the full transformation semigroup on $M$; this subsemigroup may be identified with the free semigroup $F$ on $\{s, d\}$. Hence for $f \in F$ and $u \in M, f(u)$ is a welldefined element of $M$; in fact, either $f(u) \in F_{X}(\mathbf{U})$ or $f(u)=\lambda$. For $f \in F$ we denote by $\ell(f)$ the length of $f$.

The following is a reformulation of the solution of the word problem for the free band $F_{X}(\mathbf{B})$ (see, e.g., [8]). 
Theorem 6. For $u, v \in F_{X}(\mathbf{U})$,

$$
u \rho_{\mathbf{B}} v \Leftrightarrow c(u)=c(v) \text { and } c(f(u))=c(f(v))
$$

for every $f \in F$ with $\ell(f)<|c(u)|$.

For a fully invariant congruence $\rho$ on $F_{X}(\mathbf{U})$ we define $\rho_{0}$ and $\rho_{1}$ on $F_{X}(\mathbf{U})$ by the following. For $a, b \in F_{X}(\mathbf{U})$ we have

$$
\begin{array}{ll}
a \rho_{0} b \Leftrightarrow u \rho v, & a=s(u), b=s(v) \text { for some } u, v \in F_{X}(\mathbf{U}), \\
a \rho_{1} b \Leftrightarrow u \rho v, & a=d(u), b=d(v) \text { for some } u, v \in F_{X}(\mathbf{U}) .
\end{array}
$$

Proposition 7. Let $\rho$ be a fully invariant congruence on $F_{X}(\mathbf{U})$ such that $\rho_{\mathbf{C R}} \subseteq$ $\rho \subseteq \rho_{\mathbf{B}}$. Then $\rho_{0}$ and $\rho_{1}$ are fully invariant congruences on $F_{X}(\mathbf{U})$ such that

$$
\rho_{\mathbf{C R}} \subseteq \rho_{0} \subseteq \rho_{\mathbf{B}} \text { and } \rho_{\mathbf{C R}} \subseteq \rho_{1} \subseteq \rho_{\mathbf{B}}
$$

and $\rho_{0}\left[\right.$ resp. $\left.\rho_{1}\right]$ is the greatest fully invariant congruence on $F_{X}(\mathbf{U})$ such that $\rho_{0} / \rho\left[\right.$ resp. $\left.\rho_{1} / \rho\right]$ is contained in the $\mathscr{R}-\left[\right.$ resp. $\mathscr{L}$-] relation on $F_{X}(\mathbf{U}) / \rho$.

Proof. That $\rho_{0} \subseteq \rho_{\mathbf{B}}$ and $\rho_{1} \subseteq \rho_{\mathbf{B}}$ follow immediately from Theorem 6 and the definitions of $\rho_{0}$ and $\rho_{1}$. The remaining statements now follow from Lemma 7 of [12] and the results of [13] (see also [11]).

Proposition 8. The fully invariant congruences in the sublattice $\left[\rho_{\mathbf{C R}}, \rho_{\mathbf{B}}\right]$ of $C\left(F_{X}(\mathbf{U})\right)$ commute.

Proof. For $k$ a positive integer we may consider the statement $\left(S_{k}\right)$ : If $\rho, \theta \in$ $\left[\rho_{\mathbf{C R}}, \rho_{\mathbf{R}}\right]$ and $u, v, w \in F_{X}(\mathbf{U})$ such that $u \rho v \theta w$ and $|c(u)|=k$, then there exists $t \in F_{X}(\mathbf{U})$ such that $u \theta t \rho w$.

It suffices to prove $\left(S_{k}\right)$ for every $k$. We give a proof by induction on $k$.

Let $u, v, w \in F_{X}(\mathbf{U})$ and $\rho, \theta \in\left[\rho_{\mathbf{C R}}, \rho_{\mathbf{B}}\right]$ such that $u \rho v \theta w$ and $|c(u)|=1$. Since $\rho \subseteq \rho_{\mathrm{SL}}$ and $\theta \subseteq \rho_{\mathrm{SL}}$ we have that $c(u)=c(v)=c(w)=\{x\}$ for some $x \in X$ and $\rho / \rho_{\mathbf{C R}}$ and $\theta / \rho_{\mathbf{C R}}$ induce congruences on the maximal group $\langle x\rangle$ of $F_{X}(\mathbf{C R})$. Since group congruences commute we have from $u \rho / \rho_{\mathbf{C R}} v \theta / \rho_{\mathbf{C R}} w$ that $u \theta / \rho_{\mathbf{C R}} t \rho / \rho_{\mathbf{C R}} w$ for some $t \in F_{X}(\mathbf{U})$ with $c(t)=\{x\}$. Hence $u \theta t \rho w$ and $\left(S_{1}\right)$ is satisfied.

We now assume that $k>1$ and that $\left(S_{r}\right)$ is satisfied whenever $r<k$. Assume that for $u, v, w \in F_{X}(\mathbf{U})$ and $\rho, \theta \in\left[\rho_{\mathbf{C R}}, \rho_{\mathbf{B}}\right]$ we have $u \rho v \theta w$ and $|c(u)|=k$. Again $c(u)=c(v)=c(w)$. Obviously

$$
s(u) \rho_{0} s(v) \theta_{0} s(w)
$$

with $\rho_{0}, \theta_{0} \in\left[\rho_{\mathbf{C R}}, \rho_{\mathbf{B}}\right]$ by Proposition 7. By the induction hypothesis there exists $p \in F_{X}(\mathbf{U})$ such that

$$
s(u) \theta_{0} p \rho_{0} s(w) .
$$

From $\rho_{0} \subseteq \rho_{\mathrm{SL}}$ and $\theta_{0} \subseteq \rho_{\mathrm{SL}}$ it follows that

$$
c(s(u))=c(p)=c(s(w))
$$

and so

$$
\bar{s}(u)=\bar{s}(w)=x
$$


for some $x \in X$. From (13) it now follows that

$$
(s(u) x)^{0} \theta_{0} / \rho_{\mathbf{C R}}(p x)^{0} \rho_{0} / \rho_{\mathbf{C R}}(s(w) x)^{0}
$$

in $F_{X}(\mathbf{C R})$. In a dual way we can show that there exists $q \in F_{X}(\mathbf{U})$ such that

$$
d(u) \theta_{1} q \rho_{1} d(w)
$$

and for a $y \in X$ we must have

$$
\bar{d}(u)=\bar{d}(w)=y .
$$

From (16) we then have

$$
(y d(u))^{0} \theta_{1} / \rho_{\mathbf{C R}}(y q)^{0} \rho_{1} / \rho_{\mathbf{C R}}(y d(w))^{0} .
$$

By Theorem 5.4 of [2] we have that

$$
\begin{array}{ll}
(s(u) x)^{0} \mathscr{R} u, & (s(w) x)^{0} \mathscr{R} w, \\
(y d(u))^{0} \mathscr{L} u, & (y d(w))^{0} \mathscr{L} w
\end{array}
$$

in $F_{X}(\mathbf{C R})$. From Proposition 7 we have from (15) and (18) that

$$
(s(u) x)^{0} \mathscr{R}(p x)^{0}, \quad(y d(u))^{0} \mathscr{L}(y q)^{0}
$$

in $F_{X}(\mathbf{U}) / \theta$. Therefore, using (19) and (20) we find that in $F_{X}(\mathbf{C R})$ we have

$$
u=(s(u) x)^{0} u(y d(u))^{0} \theta / \rho_{\mathbf{C R}}(p x)^{0} u(y q)^{0} .
$$

Similarly we can show from (15) and (18) that

$$
w=(s(w) x)^{0} w(y d(w))^{0} \rho / \rho_{\mathbf{C R}}(p x)^{0} w(y q)^{0}
$$

in $F_{X}(\mathbf{C R})$. By Theorem 5.4 of [2]

$$
(p x)^{0} u(y q)^{0} \mathscr{H}(p x)^{0} w(y q)^{0}
$$

in $F_{X}(\mathbf{C R})$ and by $(21),(22)$ and the fact that $u \rho / \rho_{\mathbf{C R}} v \theta / \rho_{\mathbf{C R}} w$ we also find that

$$
(p x)^{0} u(y q)^{0}\left(\left(\rho / \rho_{\mathbf{C R}}\right) \vee\left(\theta / \rho_{\mathbf{C R}}\right)\right)(p x)^{0} w(y q)^{0}
$$

in $F_{X}(\mathbf{C R})$. From (23), (24), Proposition 2(ii) and the fact that both $\rho / \rho_{\mathbf{C R}}$ and $\theta / \rho_{\mathbf{C R}}$ are contained in the $\mathscr{D}$-relation $\rho_{\mathbf{S L}} / \rho_{\mathbf{C R}}$ of $F_{X}(\mathbf{C R})$ we obain that

$$
(p x)^{0} u(y q)^{0}\left(\left(\theta / \rho_{\mathbf{C R}}\right) \circ\left(\rho / \rho_{\mathbf{C R}}\right)\right)(p x)^{0} w(y q)^{0},
$$

hence there exists $t \in F_{X}(\mathbf{U})$ such that

$$
(p x)^{0} u(y q)^{0} \theta / \rho_{\mathbf{C R}} t \rho / \rho_{\mathbf{C R}}(p x)^{0} w(y q)^{0}
$$

in $F_{X}(\mathbf{C R})$. From $(21),(22)$ and (26) we thus have

$$
u \theta / \rho_{\mathbf{C R}} t \rho / \rho_{\mathbf{C R}} w
$$

in $F_{X}(\mathbf{C R})$ and thus $u \theta t \rho w$ in $F_{X}(\mathbf{U})$. The statement $\left(S_{k}\right)$ follows. 
By the induction hypothesis the statement $\left(S_{k}\right)$ holds true for every $k \geq 1$ and the proof is complete.

For $u \in F_{X}(\mathbf{U})$ define

$$
h(u)=\bar{s}\left(s^{\ell(u)-1}(u)\right), \quad t(u)=\bar{d}\left(d^{\ell(u)-1}(u)\right),
$$

in other words, $h(u)$ and $t(u)$ are respectively the first and the last letters which appear in $u$.

In the following LNB stands for the variety of all left normal bands.

Proposition 9. Let $\rho$ be a fully invariant congruence on $F_{X}(\mathbf{U})$ such that $\rho \in$ $\left[\rho_{\mathbf{C R}}, \rho_{\mathbf{S L}}\right]$. Then

(i) if $\rho_{0} \subseteq \rho_{\mathrm{SL}}$, then $\rho_{0}$ is a fully invariant congruence on $F_{X}(\mathbf{U})$ such that $\rho \subseteq \rho_{0}$ and $\rho_{0}$ is the greatest congruence on $F_{X}(\mathbf{U})$ such that $\rho_{0} / \rho$ is contained in the $\mathscr{R}$-relation on $F_{X}(\mathbf{U}) / \rho$,

(ii) if $\rho_{0} \nsubseteq \rho_{\mathbf{S L}}$ and

$$
u \rho v \Rightarrow h(u)=h(v)
$$

for all $u, v \in F_{X}(\mathbf{U})$, then $\rho_{0} \cap \rho_{\mathbf{S L}}=\rho_{\mathbf{L N B}}$ and $\rho_{\mathbf{L N B}} / \rho$ is the $\mathscr{R}$-relation on $F_{X}(\mathbf{U}) / \rho$,

(iii) if $\rho_{0} \nsubseteq \rho_{\mathrm{SL}}$ and $u \rho v$ with $h(u) \neq h(v)$ for some $u, v \in F_{X}(\mathbf{U})$, then $\rho_{0} \cap \rho_{\mathbf{S L}}=\rho_{\mathbf{S L}}$ and $\rho_{\mathbf{S L}} / \rho$ is the $\mathscr{R}$-relation on $F_{X}(\mathbf{U}) / \rho$.

Proof. The proof follows immediately from Lemmas 5 and 6 of [16], Lemmas 6 and 7 of [12] and the results of [13] (see also [11]).

The variety of right normal bands will be denoted by RNB, the variety of normal bands by NB.

The following result is well known.

Proposition 10. For $u, v \in F_{X}(\mathbf{U})$ we have

$$
\begin{aligned}
& u \rho_{\mathbf{L N B}} v \Leftrightarrow c(u)=c(v), \quad h(u)=h(v), \\
& u \rho_{\mathbf{N B}} v \Leftrightarrow c(u)=c(v), \quad h(u)=h(v), \quad t(u)=t(v) .
\end{aligned}
$$

Proof. See, e.g., II.3 of [15].

Lemma 11. Let $\rho$ and $\theta$ be fully invariant congruences on $F_{X}(\mathbf{U})$ such that $\rho, \theta \in\left[\rho_{\mathbf{C R}}, \rho_{\mathbf{S L}}\right]$ and $u \rho v \theta w$ for some $u, v, w \in F_{X}(\mathbf{U})$ with $t(u) \neq t(v)$ and $h(u) \neq h(v)$ or $h(v) \neq h(w)$. Then there exists $t \in F_{X}(\mathbf{U})$ such that $u \theta t \rho w$.

Proof. Assume first that $h(u) \neq h(v)$ and $t(u) \neq t(v)$. By Proposition 10 we have $\operatorname{tr} \rho / \rho_{\mathbf{C R}}=\operatorname{tr} \rho_{\mathbf{S L}} / \rho_{\mathbf{C R}}$, thus $\operatorname{tr} \rho / \rho_{\mathbf{C R}} \subseteq \operatorname{tr} \theta / \rho_{\mathbf{C R}}$. By Proposition 3 it follows that $\rho \circ \theta=\theta \circ \rho$ and so there exists a $t \in F_{X}(\mathbf{U})$ with $u \theta t \rho w$.

Assume next that $h(v) \neq h(w)$ and $t(u) \neq t(v)$. Since $h(v) \neq h(w)$ then from Proposition 9(iii) we have that $u \theta / \rho_{\mathbf{C R}} w^{0} u$. Since $t(u) \neq t(v)$ we have from the dual of Proposition 9(iii) that $w u^{0} \rho / \rho_{\mathbf{C R}} w$. Invoking Theorem 5.4 of [2] we can thus write

$$
u \theta / \rho_{\mathbf{C R}} w^{0} u\left(\left(\left(\rho / \rho_{\mathbf{C R}}\right) \vee\left(\theta / \rho_{\mathbf{C R}}\right)\right) \cap \mathscr{H}\right) w u^{0} \rho / \rho_{\mathbf{C R}} w .
$$


By Proposition 2(ii) there exists $t \in F_{X}(\mathbf{U})$ such that

$$
u \theta / \rho_{\mathbf{C R}} w^{0} u \theta / \rho_{\mathbf{C R}} t \rho / \rho_{\mathbf{C R}} w u^{0} \rho / \rho_{\mathbf{C R}} w
$$

whence $u \theta t \rho w$.

Lemma 12. Let $\rho$ and $\theta$ be fully invariant congruences on $F_{X}(\mathbf{U})$ such that $\rho, \theta \in\left[\rho_{\mathbf{C R}}, \rho_{\mathbf{S L}}\right]$ and $u \rho v \theta w$ for some $u, v, w \in F_{X}(\mathbf{U})$ with $\bar{s}(u) \neq \bar{s}(v)$ and $\bar{d}(u) \neq \bar{d}(v)$. Then there exists $t \in F_{X}(\mathbf{U})$ such that $u \theta t \rho w$.

Proof. Since $\bar{s}(u) \neq \bar{s}(v)$ we have that $c(s(u)) \neq c(s(v))$, thus $\rho_{0} \nsubseteq \rho_{\mathrm{SL}}$. By Proposition 9 we then have that $\rho_{\mathrm{LNB}} / \rho$ or $\rho_{\mathrm{SL}} / \rho$ is the $\mathscr{R}$-relation on $F_{X}(\mathbf{U}) / \rho$. Dually, $\rho_{\mathbf{R N B}} / \rho$ or $\rho_{\mathbf{S L}} / \rho$ is the $\mathscr{L}$-relation on $F_{X}(\mathbf{U}) / \rho$.

Case 1. $h(u)=h(v)=h(w), t(u)=t(w)$. Using Proposition 10 we have that $u^{0} \mathscr{H} w$ in $F_{X}(\mathbf{U}) / \rho$, whence

$$
w \rho / \rho_{\mathbf{C R}} u^{0} w u^{0}\left(\left(\left(\rho / \rho_{\mathbf{C R}}\right) \vee\left(\theta / \rho_{\mathbf{C R}}\right)\right) \cap \mathscr{H}\right) u
$$

and by Proposition 2(ii) there exists a $t \in F_{X}(\mathbf{U})$ with

$$
w \rho / \rho_{\mathbf{C R}} u^{0} w u^{0} \rho / \rho_{\mathbf{C R}} t \theta / \rho_{\mathbf{C R}} u
$$

therefore $w \rho t \theta u$.

Case 2. $h(u) \neq h(v), \quad t(u)=t(w)$. By Proposition 9(iii) we have that $\rho_{\mathrm{SL}} / \rho$ is the $\mathscr{R}$-relation on $F_{X}(\mathbf{U}) / \rho$ so that by Proposition 10 we have that $u^{0} \mathscr{R} w$. Further, $t(u)=t(w)$ entails that $u^{0} \mathscr{L} w$ in $F_{X}(\mathbf{U}) / \rho$ by Proposition 10. We thus obtain $u^{0} \mathscr{H} w$ in $F_{X}(\mathbf{U}) / \rho$ and we can proceed further as in Case 1.

Case 3. $h(v) \neq h(w), t(u)=t(w)$. Here $\rho_{\mathbf{S L}} / \theta$ is the $\mathscr{R}$-relation on $F_{X}(\mathbf{U}) / \theta$, so that $w^{0} \mathscr{R} u$ in $F_{X}(\mathbf{U}) / \theta$. Further $t(u)=t(w)$ gives $w \mathscr{L} u^{0}$ in $F_{X}(\mathbf{U}) / \rho$. Hence

$$
u \theta / \rho_{\mathbf{C R}} w^{0} u\left(\left(\left(\rho / \rho_{\mathbf{C R}}\right) \vee\left(\theta / \rho_{\mathbf{C R}}\right)\right) \cap \mathscr{H}\right) w u^{0} \rho / \rho_{\mathbf{C R}} w
$$

and using Proposition 2(ii) we find a $t \in F_{X}(\mathbf{U})$ such that $u \theta t \rho w$.

Case 4. $h(u)=h(w), \quad t(u) \neq t(w)$. The case $h(u)=h(w), t(u) \neq t(v)$ deals with a situation which is the dual of Case 2 , whereas the case $h(u)=$ $h(w), t(v) \neq t(w)$ is the dual of Case 3 .

Case 5. $h(u) \neq h(w), \quad t(u) \neq t(w)$. We can discern several cases all of which are handled by Lemma 11 .

Lemma 13. Let $\rho$ and $\theta$ be fully invariant congruences on $F_{X}(\mathbf{U})$ such that $\rho, \theta \in\left[\rho_{\mathbf{C R}}, \rho_{\mathbf{S L}}\right]$ and $u \rho v \theta w$ for some $u, v, w \in F_{X}(\mathbf{U})$ with $\bar{s}(u) \neq \bar{s}(v)$ and $\bar{d}(v) \neq \bar{d}(w)$. Then there exists $t \in F_{X}(\mathbf{U})$ such that $u \theta t \rho w$.

Proof. Since $\bar{s}(u) \neq \bar{s}(v)$ we have that $c(s(u)) \neq c(s(v))$, thus $\rho_{0} \nsubseteq \rho_{\mathbf{S L}}$. By Proposition 9 we have that $\rho_{\mathbf{L N B}} / \rho$ or $\rho_{\mathrm{SL}} / \rho$ is the $\mathscr{R}$-relation on $F_{X}(\mathbf{U}) / \rho$. In a dual way we have that $\rho_{\mathbf{R N B}} / \theta$ or $\rho_{\mathrm{SL}} / \theta$ is the $\mathscr{L}$-relation on $F_{X}(\mathbf{U}) / \theta$. 
Case 1. $h(u)=h(v)=h(w), t(u)=t(w)$. Using Proposition 10 and its dual we have that $u \mathscr{L} w^{0}$ in $F_{X}(\mathbf{U}) / \theta$ and $u^{0} \mathscr{R} w$ in $F_{X}(\mathbf{U}) / \rho$. Further, since $u w^{0} \mathscr{H} u^{0} w$ in $F_{X}(\mathbf{C R})$ by Proposition 5.4 of [2], we find that

$$
u \theta / \rho_{\mathbf{C R}} u w^{0}\left(\left(\left(\rho / \rho_{\mathbf{C R}}\right) \vee\left(\theta / \rho_{\mathbf{C R}}\right)\right) \cap \mathscr{H}\right) u^{0} w \rho / \rho_{\mathbf{C R}} w .
$$

Applying Proposition 2(ii) we know that there exists $t \in F_{X}(\mathbf{U})$ with

$$
u \theta / \rho_{\mathbf{C R}} u w^{0} \theta / \rho_{\mathbf{C R}} t \rho / \rho_{\mathbf{C R}} u^{0} w \rho / \rho_{\mathbf{C R}} w,
$$

therefore $u \theta t \rho w$.

Case 2. $h(u) \neq h(v), \quad t(u)=t(w)$. By Proposition 9(iii) we have that $\rho_{\mathrm{SL}} / \rho$ is the $\mathscr{R}$-relation on $F_{X}(\mathbf{U}) / \rho$ so that by Proposition $10 u^{0} \mathscr{R} w$. Further, $t(u)=t(w)$ entails that $u \mathscr{L} w^{0}$ in $F_{X}(\mathbf{U}) / \theta$ by the dual of Proposition 10. We can proceed further as in Case 1.

Case 3. $h(v) \neq h(w), t(u)=t(w)$. Here $\rho_{\mathrm{SL}} / \theta$ is the $\mathscr{R}$-relation on $F_{X}(\mathbf{U}) / \theta$ so that $w^{0} \mathscr{R} u$ in $F_{X}(\mathbf{U}) / \theta$. Further $t(u)=t(w)$ gives $w^{0} \mathscr{L} u$ in $F_{X}(\mathbf{U}) / \theta$. Hence

$$
u \theta / \rho_{\mathbf{C R}} w^{0} u w^{0}\left(\left(\left(\rho / \rho_{\mathbf{C R}}\right) \vee\left(\theta / \rho_{\mathbf{C R}}\right)\right) \cap \mathscr{H}\right) w
$$

and using Proposition 2(ii) we find a $t \in F_{X}(\mathbf{U})$ such that $u \theta t \rho w$.

Case 4. $h(u)=h(w), t(u) \neq t(w)$. The case $h(u)=h(w), t(u) \neq t(v)$ is the dual of Case 2, whereas the case $h(u)=h(w), t(v) \neq t(w)$ is the dual of Case 3.

Case 5. $h(u) \neq h(w), t(u) \neq t(w)$. We are reduced here to cases like the ones handled by Lemma 11 .

We are now ready for the main theorem of this section.

Theorem 14. The fully invariant congruences in the sublattice $\left[\rho_{\mathbf{C R}}, \rho_{\mathbf{S L}}\right]$ of $C\left(F_{X}(\mathbf{U})\right)$ commute.

Proof. For $k$ a positive integer we now consider the statement $\left(T_{k}\right)$ : If $\rho, \theta \in$ $\left[\rho_{\mathbf{C R}}, \rho_{\mathbf{S L}}\right]$ and $u, v, w \in F_{X}(\mathbf{U})$ such that $u \rho v \theta w$ and $|c(u)|=k$, then there exists $t \in F_{X}(\mathbf{U})$ such that $u \theta t \rho w$.

It suffices to prove $\left(T_{k}\right)$ for every $k$. We give a proof by induction on $k$. The proof for $\left(T_{1}\right)$ is the same as the proof for $\left(S_{1}\right)$ in the proof of Proposition 8.

We now assume that $k>1$ and that $\left(T_{r}\right)$ is satisfied whenever $r<k$. Assume that $u, v, w \in F_{X}(\mathbf{U})$ and $\rho, \theta \in\left[\rho_{\mathbf{C R}}, \rho_{\mathbf{S L}}\right]$ such that $u \rho v \theta w$ and $|c(u)|=k$. Obviously $c(u)=c(v)=c(w)$ since $\rho, \theta \subseteq \rho_{\mathbf{S L}}$.

Case 1. $\bar{s}(u)=\bar{s}(v)=\bar{s}(w), \bar{d}(u)=\bar{d}(v)=\bar{d}(w)$. Then

$$
s(u) \rho_{0} s(v) \theta_{0} s(w), \quad c(s(u)=c(s(v))=c(s(w))
$$


so that

$$
s(u)\left(\rho_{0} \cap \rho_{\mathbf{S L}}\right) s(v)\left(\theta_{0} \cap \rho_{\mathbf{S L}}\right) s(w)
$$

where by Proposition $9 \rho_{0} \cap \rho_{\mathbf{S L}}, \theta_{0} \cap \rho_{\mathbf{S L}} \in\left[\rho_{\mathbf{C R}}, \rho_{\mathbf{S L}}\right]$. By the induction hypothesis there exists $p \in F_{X}(\mathbf{U})$ such that

$$
s(u)\left(\theta_{0} \cap \rho_{\mathbf{S L}}\right) p\left(\rho_{0} \cap \rho_{\mathbf{S L}}\right) s(w) .
$$

In a dual way we can show that there exists $q \in F_{X}(\mathbf{U})$ such that

$$
d(u)\left(\theta_{1} \cap \rho_{\mathrm{SL}}\right) q\left(\rho_{1} \cap \rho_{\mathrm{SL}}\right) d(w) .
$$

The remainder of the proof is now similar to the proof of the induction step in the proof of Proposition 8.

Case 2. $\bar{s}(u) \neq \bar{s}(v), \quad \bar{d}(u)=\bar{d}(v)=\bar{d}(w)$. Here $c(s(u)) \neq c(s(v))$ so that $\rho_{0} \nsubseteq \rho_{\mathrm{SL}}$ : either Proposition 9(ii) or Proposition 9(iii) apply. Again

$$
d(u)\left(\rho_{1} \cap \rho_{\mathbf{S L}}\right) d(v)\left(\theta_{1} \cap \rho_{\mathbf{S L}}\right) d(w)
$$

where by the dual of Proposition $9 \rho_{1} \cap \rho_{\mathbf{S L}}, \theta_{1} \cap \rho_{\mathbf{S L}} \in\left[\rho_{\mathbf{C R}}, \rho_{\mathbf{S L}}\right]$. By the induction hypothesis we can show that there exists a $q \in F_{X}(\mathbf{U})$ such that

$$
d(u)\left(\theta_{1} \cap \rho_{\mathbf{S L}}\right) q\left(\rho_{1} \cap \rho_{\mathbf{S L}}\right) d(w) .
$$

We let $y \in X$ such that

$$
\bar{d}(u)=\bar{d}(w)=y
$$

and from (32) we now have that

$$
(y d(u))^{0}\left(\theta_{1} \cap \rho_{\mathbf{S L}}\right) / \rho_{\mathbf{C R}}(y q)^{0}\left(\rho_{1} \cap \rho_{\mathbf{S L}}\right) / \rho_{\mathbf{C R}}(y d(w))^{0} .
$$

From Theorem 5.4 of [2] we have from (34) that

$$
u=u(y d(u))^{0} \theta / \rho_{\mathbf{C R}} u(y q)^{0} \mathscr{L} w(y q)^{0} \rho / \rho_{\mathbf{C R}} w(y d(w))^{0}=w .
$$

Assume first that $\rho_{0} \cap \rho_{\mathrm{SL}}=\rho_{\mathrm{LNB}}$ and $h(u)=h(w)$. We have that $\rho_{\mathrm{LNB}} / \rho$ is the $\mathscr{R}$-relation on $F_{X}(\mathbf{U}) / \rho$ by Proposition 9 . From (35) and Proposition 10 we then have that

$$
\left(u(y q)^{0}\right)^{0} \rho / \rho_{\mathbf{C R}}\left(w(y q)^{0}\right)^{0}
$$

whence

$$
w(y q)^{0} \rho / \rho_{\mathbf{C R}}\left(u(y q)^{0}\right)^{0} w(y q)^{0} .
$$

From (35), (37), Theorem 5.4 of [2] and the fact that

$$
u \rho / \rho_{\mathbf{C R}} v \theta / \rho_{\mathbf{C R}} w
$$

we find that

$$
u(y q)^{0}\left(\left(\left(\rho / \rho_{\mathbf{C R}}\right) \vee\left(\theta / \rho_{\mathbf{C R}}\right)\right) \cap \mathscr{H}\right)\left(u(y q)^{0}\right)^{0} w(y q)^{0}
$$


in $F_{X}(\mathbf{C R})$. By Proposition 2(ii) it now follows that there exists a $t \in F_{X}(\mathbf{U})$ such that

$$
u(y q)^{0} \theta / \rho_{\mathbf{C R}} t \rho / \rho_{\mathbf{C R}}\left(u(y q)^{0}\right)^{0} w(y q)^{0} .
$$

From (35), (37) and (39) we then have that $u \theta t \rho w$.

Assume next that $\rho_{0} \cap \rho_{\mathrm{SL}}=\rho_{\mathrm{SL}}$ and $h(u) \neq h(w)$. Then $\rho_{\mathrm{SL}} / \rho$ is the $\mathscr{R}$ relation on $F_{X}(\mathbf{U}) / \rho$ by Proposition 9 and from (35) we may again conclude that (36) and (37) hold. Therefore we obtain as above that there exists $t \in$ $F_{X}(\mathbf{U})$ such that $u \theta t \rho w$.

If $h(v) \neq h(w)$, then $\rho_{\mathrm{SL}} / \theta$ is the $\mathscr{R}$-relation on $F_{X}(\mathbf{U}) / \theta$ by Proposition 9 and (35), and it follows that

$$
\left(u(y q)^{0}\right)^{0} \theta / \rho_{\mathbf{C R}}\left(w(y q)^{0}\right)^{0},
$$

whence

$$
u(y q)^{0} \theta / \rho_{\mathbf{C R}}\left(w(y q)^{0}\right)^{0} u(y q)^{0} .
$$

From (35), (38), (41) and Theorem 5.4 of [2] we thus have that

$$
\left(w(y q)^{0}\right)^{0} u(y q)^{0}\left(\left(\left(\rho / \rho_{\mathbf{C R}}\right) \vee\left(\theta / \rho_{\mathbf{C R}}\right)\right) \cap \mathscr{H}\right) w(y q)^{0}
$$

in $F_{X}(\mathbf{C R})$. By Proposition 2(ii) it now follows that there exists a $t \in F_{X}(\mathbf{U})$ such that

$$
\left(w(y q)^{0}\right)^{0} u(y q)^{0} \theta / \rho_{\mathbf{C R}} t \rho / \rho_{\mathbf{C R}} w(y q)^{0} .
$$

From (35), (41) and (42) we infer that $u \theta t \rho w$.

By Proposition 9 the proof for Case 2 is now complete.

Case 3. $\bar{s}(u) \neq \bar{s}(v), \bar{d}(u) \neq \bar{d}(v)$. The existence of a $t \in F_{X}(\mathbf{U})$ such that $u \theta t \rho w$ is guaranteed by Lemma 12 .

Case 4. $\quad \bar{s}(u) \neq \bar{s}(v), \quad \bar{d}(v) \neq \bar{d}(w)$. By Lemma 13 there exists $t \in F_{X}(\mathbf{U})$ such that $u \theta t \rho w$.

Remaining cases. The proof for the remaining cases follows from the proof for Cases $1-4$ or their duals.

By the induction hypothesis the statement $\left(T_{k}\right)$ holds true for every $k \geq 1$ and the proof is complete.

Corollary 15. $\mathscr{L}(\mathbf{C R})$ is arguesian.

Proof. By Theorem 14 and [9] the sublattice [SL, CR] of $\mathscr{L}(\mathbf{C R})$ is arguesian. By Proposition 3.5 of [7], SL is a neutral element in $\mathscr{L}(\mathbf{C R})$, so that $\mathscr{L}(\mathbf{C R})$ is a subdirect product of $[\mathbf{S L}, \mathbf{C R}]$ and the two element lattice $[\mathbf{T}, \mathbf{S L}]$. It follows that $\mathscr{L}(\mathbf{C R})$ is arguesian.

Remark 16. From [1] or [4] or [5] it follows that the lattice $\mathscr{L}(\mathbf{B})$ of all band varieties is distributive. From [18] we know that $\mathbf{B}$ is a neutral element in $\mathscr{L}(\mathbf{C R})$ so that $\mathscr{L}(\mathbf{C R})$ is a subdirect product of $[\mathbf{B}, \mathbf{C R}]$ and $\mathscr{L}(\mathbf{B})$. Now $[\mathbf{B}, \mathbf{C R}]$ is arguesian by Proposition 8 . Thus the fact that $\mathscr{L}(\mathbf{C R})$ is arguesian 
follows from Proposition 8 and the results of $[1,4,5]$ and the fact that $\mathbf{B}$ is neutral in $\mathscr{L}(\mathbf{C R})$.

Remark 17. In [6], Haiman established an infinite sequence of identities satisfied by linear lattices (i.e. lattices representable by commuting equivalences) but not by all arguesian lattices. From our results it follows that $\mathscr{L}(\mathbf{C R})$ satisfies all these identities.

Problem. Theorem 4 and Theorem 14 support the following conjecture: if $S$ is a completely regular semigroup, $\mathscr{D}$ the least semilattice congruence on $S$ and $\rho, \theta$ fully invariant congruences on $S$ such that $\rho, \theta \subseteq \mathscr{D}$, then $\rho \circ \theta=\theta \circ \rho$.

\section{REFERENCES}

1. A. P. Birjukov, Varieties of idempotent semigroups, Algebra i Logika 9 (1970), 255-273. (Russian)

2. A. H. Clifford, The free completely regular semigroup on a set, J. Algebra 59 (1979), 434-451.

3. A. H. Clifford and G. B. Preston, The algebraic theory of semigroups, Vols. I, II, Math. Surveys, no. 7, Amer. Math. Soc., Providence, R.I. 1961, 1967.

4. C. F. Fennemore, All varieties of bands. I, II, Math. Nachr 48 (1971), 237-252, 253-262.

5. J. A. Gerhard, The lattice of equational classes of idempotent semigroups, J. Algebra $\mathbf{1 5}$ (1970), 195-224.

6. M. D. Haiman, Arguesian lattices which are not linear, Bull. Amer. Math. Soc. (N.S.) 16 (1987), 121-123.

7. T. E. Hall and P. R. Jones, On the lattice of varieties of bands of groups, Pacific J. Math. 91 (1980), 327-337.

8. J. M. Howie, An introduction to semigroup theory, Academic Press, London, 1976.

9. B. Jónson, On the representation of lattices, Math. Scand. 1 (1953), 193-206.

10. G. Lallement, Congruences et équivalences de Green sur un demi-groupe régulier, C. R. Acad. Sci. Paris Sér. A 262 (1966), 613-616.

11. F. Pastijn, Congruences on regular semigroups-A survey, Proc. 1984 Marquette Conference on Semigroups, Marquette University, Milwaukee, 1985, pp. 159-175.

12. 24-42.

13. F. Pastijn and M. Petrich, Congruences on regular semigroups, Trans. Amer. Math. Soc. 295 (1986), 607-633.

14. M. Petrich, Introduction to semigroups, Merrill, Columbus, 1973.

15. __ Lectures in semigroups, Akademie-Verlag, Berlin, 1977.

16. L. Polák, On varieties of completely regular semigroups. I, Semigroup Forum 32 (1985), 97-123.

17. $\ldots$, On varieties of completely regular semigroups. II, Semigroup Forum 36 (1987), 253-284.

18. P. G. Trotter, Subdirect decompositions of the lattice of varieties of completely regular semigroups, Bull. Austral. Math. Soc. 39 (1989), 343-351.

Department of Mathematics, Statistics and Computer Science, Marquette UniverSity, MiLwaUkeE, Wisconsin 53233 